Draft Version June 30, 2021

Preprint typeset using $\mathrm{IAT}_{\mathrm{E}} \mathrm{X}$ style emulateapj v. 5/2/11

\title{
FIRST CONNECTION BETWEEN COLD GAS IN EMISSION AND ABSORPTION: CO EMISSION FROM A GALAXY-QUASAR PAIR
}

\author{
Marcel Neeleman ${ }^{1}$, J. Xavier Prochaska ${ }^{1}$, Martin A. Zwaan ${ }^{2}$, Nissim Kanekar ${ }^{3}$, Lise Christensen $^{4}$, Miroslava \\ Dessauges-Zavadsky ${ }^{5}$, Johan P.U. Fynbo ${ }^{4}$, Eelco van Kampen ${ }^{2}$, Palle Møller $^{2}$, Tayyaba Zafar ${ }^{6}$ \\ Draft version June 30, 2021
}

\begin{abstract}
We present the first detection of molecular emission from a galaxy selected to be near a projected background quasar using the Atacama Large Millimeter/submillimeter Array (ALMA). The ALMA detection of $\mathrm{CO}(1-0)$ emission from the $z=0.101$ galaxy toward quasar PKS 0439-433 is coincident with its stellar disk and yields a molecular gas mass of $M_{\mathrm{mol}} \approx 4.2 \times 10^{9} M_{\odot}$ (for a Galactic COto- $\mathrm{H}_{2}$ conversion factor), larger than the upper limit on its atomic gas mass. We resolve the $\mathrm{CO}$ velocity field, obtaining a rotational velocity of $134 \pm 11 \mathrm{~km} \mathrm{~s}^{-1}$, and a resultant dynamical mass of $\geq 4 \times 10^{10} \mathrm{M}_{\odot}$. Despite its high metallicity and large molecular mass, the $z=0.101$ galaxy has a low star formation rate, implying a large gas consumption timescale, larger than that typical of late-type galaxies. Most of the molecular gas is hence likely to be in a diffuse extended phase, rather than in dense molecular clouds. By combining the results of emission and absorption studies, we find that the strongest molecular absorption component toward the quasar cannot arise from the molecular disk, but is likely to arise from diffuse gas in the galaxy's circumgalactic medium. Our results emphasize the potential of combining molecular and stellar emission line studies with optical absorption line studies to achieve a more complete picture of the gas within and surrounding high-redshift galaxies. Subject headings: galaxies: kinematics and dynamics — galaxies: ISM — ISM: molecules -submillimeter: ISM - quasars: absorption lines - quasars: individual
\end{abstract} (PKS0439-433)

\section{INTRODUCTION}

A crucial link in our understanding of galaxy formation and evolution is the recognition of the pivotal role that gas plays in the process. In a $\Lambda$ cold dark matter cosmology, initial small dark matter overdensities merge to become large overdensities in which galaxies can grow. However, it is the baryonic component in and surrounding this nascent galaxy, in the form of gas, that shapes and nurtures the galaxy, feeding its growth, and acting as the fuel for star formation. Studies of gas in and around galaxies are hence critical to understand galaxy evolution.

Absorption studies have long provided the best route toward probing the gaseous environs of galaxies at high redshifts. Using samples of galaxies with a nearby projected background quasar (i.e. "quasar-galaxy pairs"), the circumgalactic medium (CGM) of different galaxy populations can be probed (e.g., Hennawi et al. 2006; Stocke et al. 2013; Tumlinson et al. 2013; Bordoloi et al. 2014). These studies find that the CGM extends out

\footnotetext{
${ }^{1}$ Department of Astronomy \& Astrophysics, UCO/Lick Observatory, University of California, 1156 High Street, Santa Cruz, CA 95064, USA; marcel@ucsc.edu

2 European Southern Observatory, Karl-Schwarzschild-strasse 2, D-85748 Garching bei München, Germany

${ }^{3}$ Swarnajayanti Fellow; National Centre for Radio Astrophysics, Tata Institute of Fundamental Research, Pune 411007, India

${ }^{4}$ Dark Cosmology Centre, Niels Bohr Institute, University of Copenhagen, Juliane Maries Vej 30, DK-2100 Copenhagen, Denmark

${ }^{5}$ Observatoire de Genève, Université de Genève, $51 \mathrm{Ch}$. des Maillettes, 1290 Sauverny, Switzerland

${ }^{6}$ Australian Astronomical Observatory, P.O. Box 915, North Ryde, NSW 1670, Australia
}

to at least $100 \mathrm{kpc}$ around the most massive galaxies (Prochaska et al. 2011), contains a large fraction of both cool and hot ionized gas (Werk et al.|2014), and is metalenriched (e.g. Simcoe et al. 2006; Lau et al. 2016). Conversely, selecting samples of strong absorbers through direct quasar spectroscopy allows for the study of the gas in and surrounding more representative galaxies, as the usual biases in identifying galaxy samples are not employed here (e.g., Wolfe et al. 2005).

As part of an Atacama Large Millimeter/submillimeter Array (ALMA) program to study molecular gas in high-metallicity, absorption-selected galaxies, we targeted the strong metal-line absorber at $z=0.101$ toward PKS0439-433 (Petitjean et al. 1996). This supersolar metallicity absorber is associated with a spiral galaxy, at a projected distance of $7.3 \mathrm{kpc}$ to the quasar (Petitiean et al. 1996; Chen et al. 2005), providing an ideal quasar-galaxy pair for studying the molecular gas in the galaxy, as well as its CGM. Besides providing a detailed characterization of the host galaxy, our ALMA observations indicate that the absorber is probing a molecular gas component of galaxies that is essentially impossible to observe in emission. Our study thus highlights the invaluable information that can be obtained by studying the galactic counterparts of absorbers in constraining how galaxies form and evolve.

\section{THE $Z=0.101$ ABSORBER TOWARD PKS 0439-433}

The $z=0.101$ absorber toward PKS 0439-433, has been studied in detail in multiple wavebands, using both imaging and spectroscopy. The H I column density of the absorber has been measured from the Lyman$\alpha$ absorption line to be $\log \left[N_{\mathrm{H} \text { I }} / \mathrm{cm}^{-2}\right]=19.63 \pm 0.08$ 
2001).

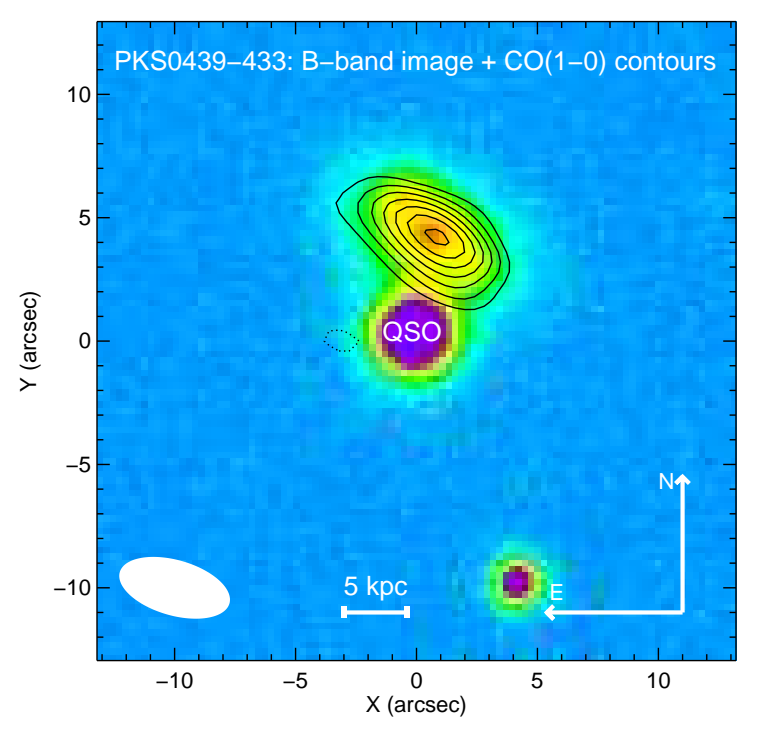

Figure 1. Integrated $\mathrm{CO}(1-0)$ emission, in contours, overlaid on a B-band image of the field surrounding PKS 0439-433 taken by Chen et al. (2005). The outermost CO contour is at $3 \sigma$ significance, with each successive contour increasing by $\sqrt{2}-\sigma$. It is clear that the $\mathrm{CO}(1-0)$ emission arises from the disk of the $z=0.101$ spiral galaxy.

(Muzahid et al. 2015; Som et al. 2015). The detection of several low-ionization metal lines, in particular SII, indicates that the absorber has two main absorption complexes at $z_{1}=0.10094$ and $z_{2}=0.10119$, with a super-solar gas-phase metallicity, $[\mathrm{S} / \mathrm{H}]=0.28 \pm 0.08$ (Dutta et al. 2015). These two main absorption components are also seen in CaII and Na I absorption toward the quasar (Richter et al. 2011).

The system furthermore shows ultraviolet $\mathrm{H}_{2}$ absorption, with $\mathrm{H}_{2}$ column density $\approx(4.1 \pm 0.5) \times 10^{16} \mathrm{~cm}^{-2}$ (Muzahid et al. 2015), and also has a tentative $(3.3 \sigma)$ detection of $\mathrm{HI} 21 \mathrm{~cm}$ absorption, yielding a low spin temperature, $\approx 90 \mathrm{~K}$ (Kanekar et al. 2001; Dutta et al. 2015), although the H I $21 \mathrm{~cm}$ feature does not coincide in velocity with the metal lines.

Optical spectroscopy of the $z=0.101$ spiral galaxy has identified multiple emission lines, including $\mathrm{H} \alpha, \mathrm{H} \beta$, [O II], etc. (Petitiean et al. 1996; Chen et al. 2005). Chen et al. (2005) used slit spectroscopy of the $\mathrm{H} \beta$ line to find that the galaxy's velocity field is consistent with arising from a rotating disk at an inclination of $58^{\circ} \pm 5^{\circ}$. The $\mathrm{H} \alpha$ luminosity was used by Dutta et al. (2015) to estimate a star formation rate (SFR) of $0.53 M_{\odot} \mathrm{yr}^{-1}$ based on a Salpeter IMF, without including corrections due to slit-losses of the $\mathrm{H} \alpha$ emission.

Finally, the stellar mass of the spiral galaxy has been estimated to be $\log \left[M_{\star} / M_{\odot}\right]=10.01 \pm 0.02$, via a fit of spectral energy distribution models to the optical photometry (Christensen et al. 2014). This also yielded an estimate for the SFR of $\approx 1.5 M_{\odot} \mathrm{yr}^{-1}$ without correcting for intrinsic extinction. The non-detection of H I $21 \mathrm{~cm}$ emission from the galaxy yields a 3- $\sigma$ upper limit of $3 \times 10^{9} M_{\odot}$ on its atomic gas mass (Kanekar et al.

\section{ALMA OBSERVATIONS}

We used ALMA band 3 receivers to carry out a deep search for redshifted $\mathrm{CO}(1-0)$ emission from the $z=$ 0.101 galaxy on UT 2014 December 25. A compact antenna configuration resulted in an angular resolution of $\sim 2^{\prime \prime}$, corresponding to $\approx 3.7 \mathrm{kpc}$ at $z=0.101$. One of the four spectral windows was centered at the redshifted $\mathrm{CO}(1-0)$ line frequency of $104.7 \mathrm{GHz}$, while the three other spectral windows were used for continuum observations of the field. QSO J0439-4522 and Uranus were used as gain and amplitude calibrators, respectively.

The data were analyzed following standard procedures in the Common Astronomy Software Applications package (CASA; McMullin et al. 2007). The continuum image was made by combining the three continuum spectral windows, resulting in a root mean square (RMS) noise of $61 \mu \mathrm{Jy}$. The quasar was detected at high signal-to-noise ratio in this image, allowing for self-calibration of the visibility data by performing one round of phase only and one round of phase and amplitude calibration. After selfcalibration, the final spectral cube covering the $\mathrm{CO}(1-0)$ line was made with a velocity resolution of $20 \mathrm{~km} \mathrm{~s}^{-1}$, using Briggs weighting with a robust factor of 0.5 and the clean routine. The synthesized beam size of the cube was $4.28^{\prime \prime} \times 1.96^{\prime \prime}$, at a position angle of $73.85^{\circ}$. The final RMS noise of the data cube is $0.76 \mathrm{mJy} \mathrm{beam}^{-1}$ per $20 \mathrm{~km} \mathrm{~s}^{-1}$ channel.

\section{RESULTS}

A search for line emission in the spectral cube yields a strong $(12 \sigma)$ detection of emission, at a frequency corresponding to $z=0.10100$ for the $\mathrm{CO}(1-0)$ line. Figure 1 shows the integrated $\mathrm{CO}(1-0)$ emission overplot-
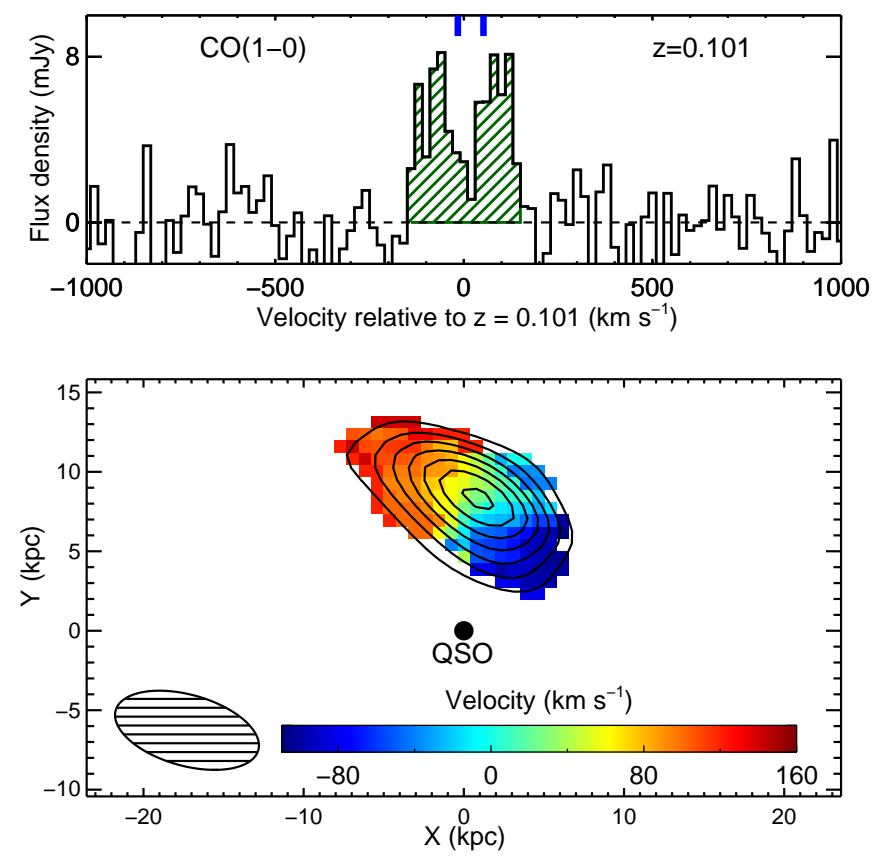

Figure 2. Top: integrated flux density as a function of the velocity, where $v=0 \mathrm{~km} \mathrm{~s}^{-1}$ corresponds to $z=0.10100$. Blue tick marks indicate the velocity centroid of the two absorption components. Bottom: velocity field of the $\mathrm{CO}(1-0)$ emission from the $z=0.101$ spiral galaxy. See the text for a discussion. 

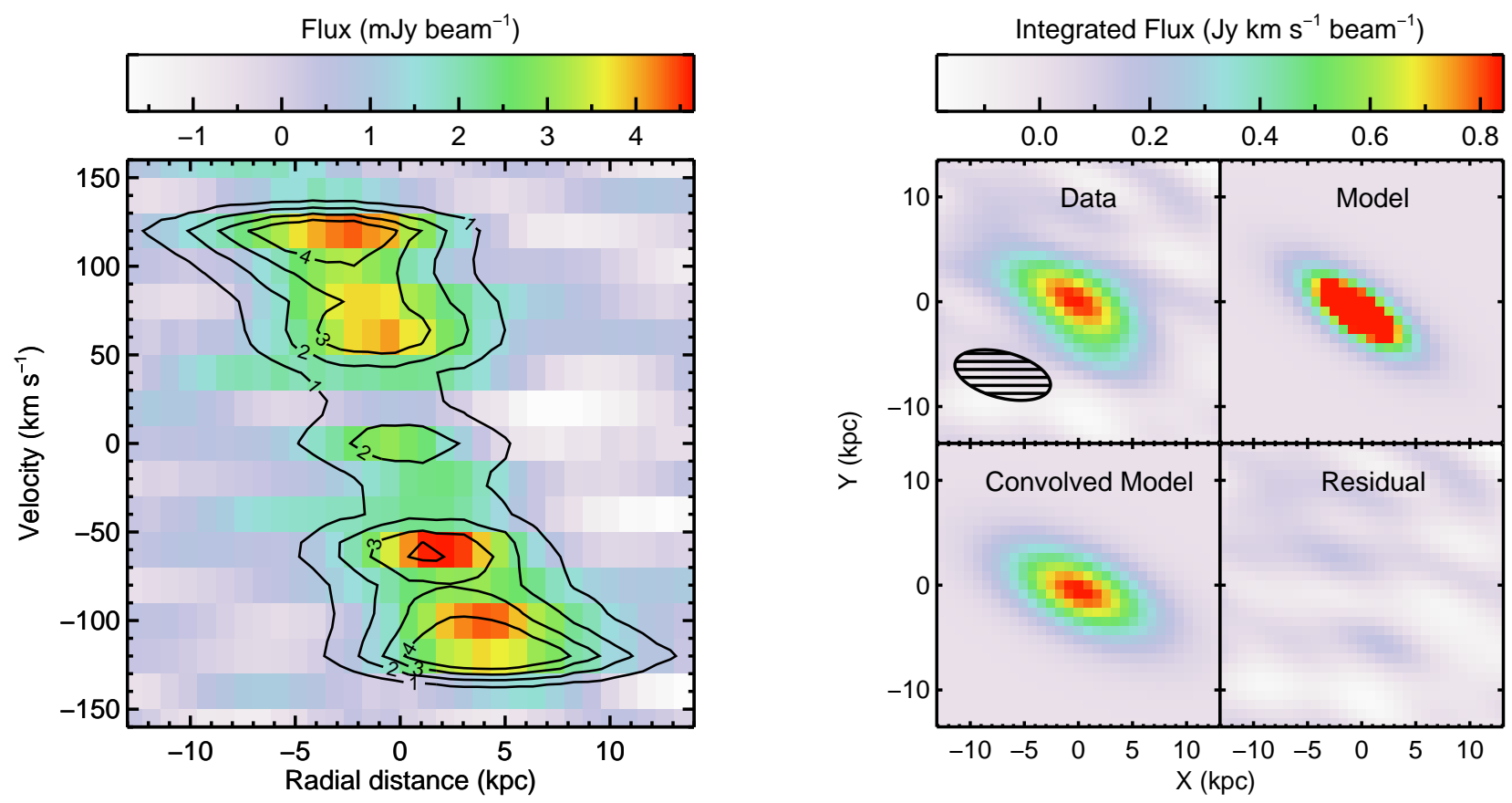

Figure 3. Left: position-velocity $(p-v)$ diagram of the $\mathrm{CO}(1-0)$ line, for a cut along the major axis of the galaxy. Overplotted on the data, in contours, is the best-fit model from the Monte Carlo Markov Chain method described in the text convolved with the ALMA beam. Right: comparison of the integrated $\mathrm{CO}(1-0)$ line emission for the data, model, and convolved model. The residual and $p-v$ diagrams show that the simple infinitely thin disk-model describes the data well.

ted on the B-band optical image of Chen et al. (2005). The $\mathrm{CO}$ emission is centered on the spiral galaxy previously associated with the absorber (Petitiean et al. 1996; Chen \& Lanzetta 2003), with excellent agreement in both the alignment and the spatial extent of the $\mathrm{CO}$ and the optical emission. We obtain an integrated $\mathrm{CO}(1-0)$ flux density of $1.46 \pm 0.07 \mathrm{Jy} \mathrm{km} \mathrm{s}^{-1}$, which results in a line luminosity of $L_{\mathrm{CO}}^{\prime}=7.0 \pm 0.3 \times 10^{8} \mathrm{~K} \mathrm{~km} \mathrm{~s}^{-1} \mathrm{pc}^{2}$, or $L_{\mathrm{CO}}=3.4 \pm 0.2 \times 10^{4} L_{\odot}$.

No $\mathrm{CO}(1-0)$ absorption was detected toward the quasar, yielding a $3 \sigma$ upper limit of $\approx 0.027 \mathrm{~km} \mathrm{~s}^{-1}$ on the integrated line opacity. Assuming a line excitation temperature of $\approx 10 \mathrm{~K}$ and a Galactic CO-to$\mathrm{H}_{2}$ conversion factor typical of diffuse/translucent clouds $\left(\mathrm{N}(\mathrm{CO}) / \mathrm{N}\left(\mathrm{H}_{2}\right) \approx 3 \times 10^{-6}\right.$; Burgh et al. 2007), we obtain $\mathrm{N}\left(\mathrm{H}_{2}\right)<6 \times 10^{19} \mathrm{~cm}^{-2}$, consistent with the estimated $\mathrm{H}_{2}$ column density from the ultraviolet lines.

The integrated $\mathrm{CO}(1-0)$ emission profile is displayed in the top panel of Figure 2 Furthermore, the emission is spatially resolved even in ALMA's compact configuration, allowing an estimate of the velocity field of the galaxy. The bottom panel of Figure 2 shows the first velocity moment of the $\mathrm{CO}(1-0)$ emission obtained by using the immoments function in CASA, and by only including emission detected at greater than $3 \sigma$ significance. Both the 'spider' pattern in this figure and the 'double-horned' feature in the integrated line profile indicate the $\mathrm{CO}(1-0)$ emission arises from a rotating disk (e.g., De Blok \& Walter 2014), strongly suggesting that the emission originates from the interstellar medium of the spiral galaxy.

To quantitatively describe the results, one must take into account several observational effects, most importantly beam smearing and inclination. To disentangle these effects, we have developed a custom Monte Carlo
Markov Chain method, which we describe in the next section.

\subsection{Modeling the $\mathrm{CO}(1-0)$ Emission}

We model the $\mathrm{CO}(1-0)$ emission from the $z=0.101$ galaxy by an infinitely thin, exponential disk. For simplicity, we assume a flat rotation curve, such that the rotational velocity of the molecular gas does not depend on radius. We note that the intrinsic velocity dispersion for this model is zero, the implications of which we will discuss later.

To account for beam smearing, we adopt the following procedure. First, we construct a model data cube by modeling the average $\mathrm{CO}$ emission as an exponential disk of the form

$$
I\left(\mathrm{Jy}_{\text {beam }}{ }^{-1}\right)=I_{0} e^{\left(-R / R_{D}\right)},
$$

where $I_{0}$ is the intensity of the emission line at the galaxy's center and $R_{D}$ is the turnover radius. The disk's orientation is determined by its inclination $(i)$ and the position angle of the major axis with respect to north $(\alpha)$. The galaxy's center in the data cube is described by three coordinates, two spatial, and one frequency. Finally, the rotation of the molecular disk is modeled by a constant rotational velocity along the disk $\left(v_{\max }\right)$.

The resultant model data cube is then convolved with the ALMA beam and compared with the data set. We apply a Monte Carlo Markov Chain method using the Metropolis-Hastings algorithm to determine the posterior probability distribution function of each parameter of the model. During this process, we remove the 'burnin' phase by discarding the first $40 \%$ of the chain. We check for convergence by choosing five different starting points and running five different iterations.

From the residuals in Figure 3, we can see that this 
simple model describes the data quite well. The residuals show no evidence of sub-structures, such as warped disks, although higher- resolution data would be required to confirm this. For the model, we find an inclination angle of $67_{-5}^{+6}$ degrees, with a position angle of $56^{\circ} \pm 5^{\circ}$, and a rotational velocity of $134_{-11}^{+8} \mathrm{~km} \mathrm{~s}^{-1}$. These values are in good agreement with those derived from the $\mathrm{H} \beta$ line (Chen et al. 2005).

\subsection{Kinematic Structure of the Molecular Gas}

As shown previously, the $\mathrm{CO}(1-0)$ emission profile is consistent with arising from a rotating molecular disk. To assess if the molecular gas seen in absorption also arises from this disk, we can calculate the rotational velocity needed to produce the velocity shifts seen in the absorption components. This is shown in Figure 4 It is clear that the velocity of absorption component 1 is consistent with arising from the disk. However, the dominant molecular gas resides in component 2, whose velocity is inconsistent with arising from an extended molecular disk.

Therefore, we conclude that absorption component 2 is unlikely to probe the same molecular disk seen in emission and must instead be probing molecular gas in either the CGM, a strong metal-rich outflow, or a nearby faint dwarf galaxy. The high metallicity of the gas favors the first two scenarios as a clear correlation between metallicity and mass exists, both for galaxies (e.g., Tremonti et al. 2004) and absorbers (Ledoux et al.|2006; Prochaska et al. 2008; Kanekar et al. 2009; Møller et al.

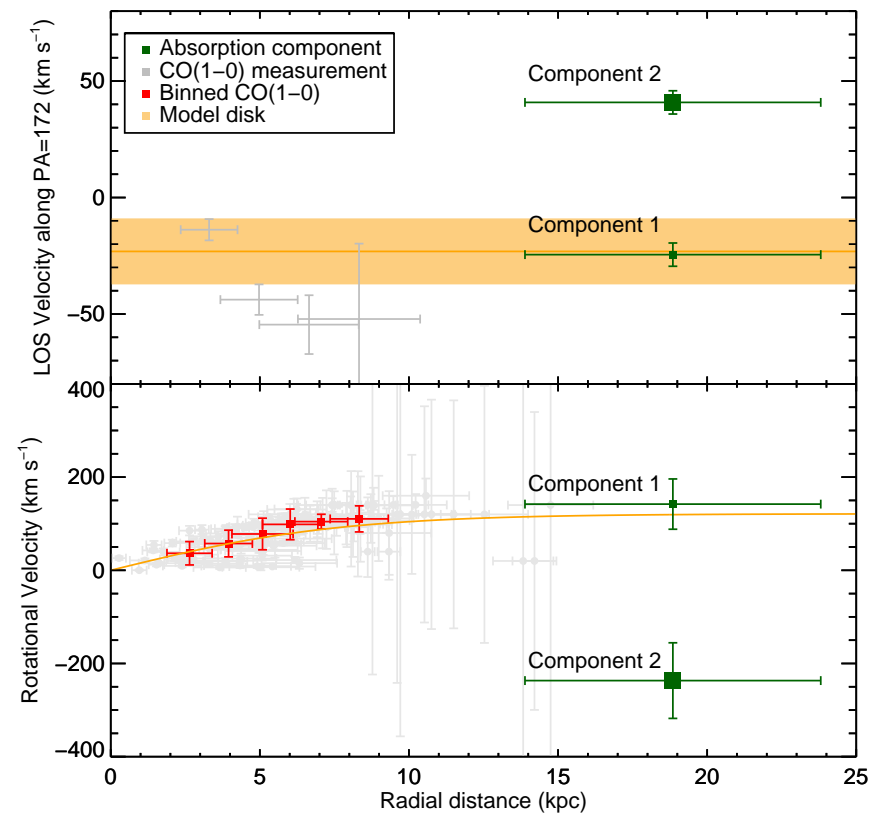

Figure 4. Top: line of sight (LOS) velocity component along PA = $172^{\circ}$. The gray points are measurements of the LOS velocity of the $\mathrm{CO}$ emission, whereas the orange shaded region is the LOS velocity along this PA for the best-fit model. The green data points are the two absorption components. Bottom: rotation curve of the molecular disk. The $\mathrm{CO}(1-0)$ emission profile (with raw and binned measurements in gray and red, respectively) is well described by a model with constant rotational velocity (orange). Both plots show that the main absorption complex (component 2) is inconsistent with coming from an extended molecular disk.
2013; Neeleman et al. 2013; Christensen et al. 2014). The velocity measured from the gas results in very modest outflow speeds $\left(\lesssim 25 \mathrm{~km} \mathrm{~s}^{-1}\right)$, blurring the distinction between outflow and CGM. We therefore assert that the dominant $\mathrm{H}_{2}$ absorption component stems from the CGM of the galaxy, in agreement with the results of Dutta et al. (2015), based on photoionization modeling of $\mathrm{H}_{2}$ and $\mathrm{NaI}$ of the absorber.

\subsection{The Gas and Dynamical Mass of the Galaxy}

The measured $\mathrm{CO}(1-0)$ line luminosity can be converted to a molecular gas mass by assuming a COto- $\mathrm{H}_{2}$ conversion factor, $\alpha_{\mathrm{CO}}$, and including a correction for helium. For this purpose, we will assume $\alpha_{\mathrm{CO}}=4.3 M_{\odot}\left(\mathrm{K} \mathrm{km} \mathrm{s}^{-1} \mathrm{pc}^{2}\right)^{-1}$, as recommended by Bolatto et al. (2013) for normal galaxies of solar metallicity. This implies a total molecular gas mass of $M_{\mathrm{mol}}=$ $4.2 \pm 0.2 \times 10^{9} M_{\odot}$, which is higher than the $3 \sigma$ upper limit on the neutral atomic gas mass $\left(\leq 3 \times 10^{9} M_{\odot}\right.$; Kanekar et al. 2001). The mass ratio of molecular to atomic gas is $\geq 1.3$, placing this galaxy at the top end of the distribution compared to other late-type galaxies of comparable stellar mass (e.g., Lisenfeld et al.|2011; Saintonge et al. 2011; Boselli et al. 2014; Bothwell et al. 2014; Jiang et al. 2015).

The molecular gas fraction relative to stars $\left(M_{\mathrm{mol}} / M_{\star}\right)$ is also quite high, $\approx 0.4$; for comparison, Boselli et al. (2014) obtain a typical molecular gas fraction of $\approx 0.1$ for late-type galaxies at low redshifts, although again this fraction falls within the expected spread of latetype galaxies. Combining the total molecular gas mass with the estimated SFR of $1.5 M_{\odot} \mathrm{yr}^{-1}$ yields a gas consumption timescale of $\approx 2.8 \mathrm{Gyr}$, larger than the typical gas consumption timescales $(\approx 1 \mathrm{Gyr})$ seen in latetype galaxies of similar stellar mass (e.g., Boselli et al. 2014). The low SFR suggests that the molecular gas in the galaxy may be dominated by diffuse gas rather than by dense giant molecular clouds. Observations of dense gas tracers such as $\mathrm{HCN}$ or $\mathrm{HCO}^{+}$would be of much interest in this regard.

One can also use the rotation velocity to infer the dynamical mass of the galaxy, for an assumed dark matter density profile. Ignoring the details of the density profile, the dynamical mass can be roughly estimated as $M_{\text {dyn }} \sim V^{2} R / G$, where $V$ is the rotation velocity and $R$ is the radius of the gas disk. In the present case, we have $V \approx 134 \mathrm{~km} \mathrm{~s}^{-1}$ and $R \approx 10 \mathrm{kpc}$, yielding $M_{\text {dyn }} \gtrsim 4 \times 10^{10} M_{\odot}$. This is likely to be a lower limit to the dynamical mass as the radius $R$ has been estimated from the molecular disk (whose size is similar to that of the stellar disk), which is likely significantly less extended than the atomic gas (e.g., Cayatte et al. 1994).

\subsection{Surface Mass Density of the Molecular Gas}

Our exponential disk model yields an estimate of the $\mathrm{CO}$ intensity per unit area as a function of radius, which can be converted to a surface mass density with the assumption that the intensity scales with the mass of molecular gas, i.e. $\mathrm{M}_{\mathrm{H}_{2}}=\alpha_{\mathrm{CO}} L_{\mathrm{CO}}^{\prime}$. The molecular absorption yields an additional constraint on the surface mass density at the position where the line of sight crosses the disk plane.

Figure 5 shows that the molecular gas seen in absorption does not fit a simple extrapolation of the exponential 
disk model. The extrapolation of the model to where the sightline crosses the plane of the disk $(\approx 20 \mathrm{kpc})$ predicts an average surface density several orders of magnitude higher than that measured in absorption. This indicates that the absorption components are tracing a more diffuse molecular gas than that responsible for the $\mathrm{CO}(1-0)$ emission.

We can estimate the total amount of molecular gas probed by the absorption component, if we make the following three assumptions: (i) the covering fraction of this gas is small, $<10 \%$ (this is corroborated by the paucity of $\mathrm{H}_{2}$ absorption in absorption-selected galaxies; e.g., Ledoux et al. 2003; Noterdaeme et al. 2008); (ii) the measured $\mathrm{H}_{2}$ column density toward PKS 0439-433 is representative of the whole distribution of gas; and (iii) only gas within a sphere of $20 \mathrm{kpc}$ of the galaxy is considered. Under these assumptions, we find that the absorbing molecular component contains $\lesssim 10^{5} M_{\odot}$ of gas. This is negligible in comparison to the mass of the galaxy and would be undetectable in emission at cosmological distances even with ALMA.

The presence of molecular gas in the CGM indicates that a small fraction of the gas is dense and cold enough for molecular gas to be shielded from dissociating radiation. Such gas could be formed elsewhere and survived the transport into the CGM as is the favored explanation for $\mathrm{H}_{2}$ gas seen locally in high-velocity clouds (e.g., Sembach et al. 2001), or could have formed in situ onto dust grains. A detailed analysis of these two scenarios is beyond the scope of this paper.

Although the molecular gas probed by the absorption only contains a tiny fraction of the total molecular content of the galaxy, it may be directly responsible for fueling star formation in the galaxy and may hence play a crucial role in the galaxy's evolution. Several observational attributes bolster this claim: (i) the absorbing gas has been heavily enriched by the host galaxy, (ii) its kinematics indicate that the gas is bound to the galaxy, and (iii) it is relatively cold and may hence more readily fuel star formation.

\section{CONCLUDING REMARKS}

We have used ALMA to obtain the first detection of molecular emission from a galaxy selected to be nearby a projected background quasar. The detected $\mathrm{CO}(1-0)$ emission yields a molecular gas mass of $\approx 4.2 \times 10^{9} M_{\odot}$, larger than the upper limit on the atomic gas mass in this system. The large inferred gas depletion time suggests that the molecular gas is mostly in a diffuse phase, rather than in dense giant molecular clouds. By combining emission and absorption studies, we find that the strongest absorption component does not arise in the disk of the galaxy, but instead is likely to stem from a diffuse CGM that is nearly impossible to detect directly through line emission or optical imaging. Because of its metal enrichment and velocity structure, we assert this CGM gas has been enriched by the galaxy and could be fueling star formation in the galaxy in the future. This study highlights the synergy between molecular emission studies and absorption studies to evaluate the cycle of gas within and surrounding galaxies.

We would like to thank H.-W. Chen for providing the

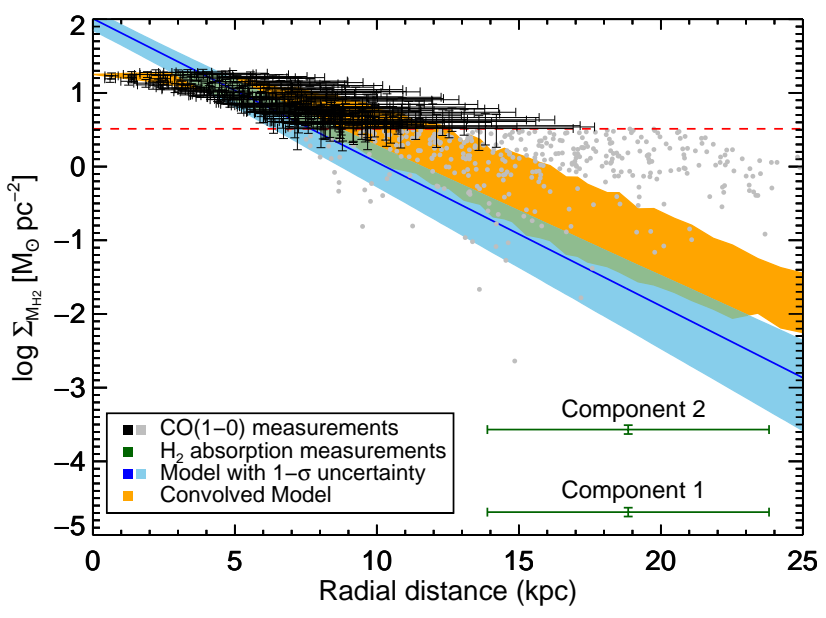

Figure 5. Surface density of molecular hydrogen as a function of radius. The red dashed line indicates the $2 \sigma$ detection limit of the ALMA data. Integrated $\mathrm{CO}(1-0)$ emission features of $\geq$ $2-\sigma$ significance are plotted in black with associated error bars, whereas those below this limit are shown in gray. The blue line marks the exponential disk model used for the MCMC analysis. This model is convolved with the ALMA beam (orange region), showing the excellent agreement between the model fit and the data. The surface densities computed from the $\mathrm{H}_{2}$ absorption lines are plotted at the distance the line of sight crosses the plane of the disk, showing that neither absorption component trace the same molecular phase responsible for the $\mathrm{CO}(1-0)$ emission.

B-band image of PKS 0439-433. M.N. and J.X.P. acknowledge support from NSF award AST-1109452, N.K. from the Department of Science and Technology through a Swarnajayanti Fellowship (DST/SJF/PSA-01/201213), and L.C. from YDUN grant DFF 4090-00079. The research leading to these results has received funding from the European Research Council under the European Union's Seventh Framework Program (FP7/20072013)/ERC Grant agreement No. EGGS-278202.

This paper makes use of the following ALMA data: ADS/JAO.ALMA\#2013.1.01178.S. ALMA is a partnership of ESO (representing its member states), NSF (USA) and NINS (Japan), together with NRC (Canada) and NSC and ASIAA (Taiwan) and KASI (Republic of Korea), in cooperation with the Republic of Chile. The Joint ALMA Observatory is operated by ESO, AUI/NRAO and NAOJ. The National Radio Astronomy Observatory is a facility of the National Science Foundation operated under cooperative agreement by Associated Universities, Inc.

\section{REFERENCES}

Bolatto, A., Wolfire, M., \& Leroy, A. 2013, ARA\&A, 51, 207 Bordoloi, R., Tumlinson, J., Werk, J., et al. 2014, ApJ, 796, 136 Boselli, A., Cortese, L., Boquien, M., et al. 2014, A\&A, 564, 66 Bothwell, M. S., Wagg, J., Cicone, C., et al. 2014, MNRAS, 445, 2599

Burgh, E. B., France, K., \& McCandliss, S. 2007, ApJ, 658, 446

Cayatte, V., Kotanyi, C., Balkowski, C., \& Van Gorkom, J. 1994, AJ, 107, 1003

Chen, H.-W., Kennicutt, R., \& Rauch, M. 2005, ApJ, 620, 703

Chen, H.-W., \& Lanzetta, K. 2003, ApJ, 597, 706

Christensen, L., Møller, P., Fynbo, J., \& Zafar, T. 2014, MNRAS, 445,225

De Blok, W., \& Walter, F. 2014, AJ, 147, 96

Dutta, R., Srianand, R., Muzahid, S., et al. 2015, MNRAS, 448, 3718 
Hennawi, J. F., Prochaska, J. X., Burles, S., et al. 2006, ApJ, 651, 61

Jiang, X.-J., Wang, Z., Gu, Q., Wang, J., \& Zhang, Z.-Y. 2015, ApJ, 799, 92

Kanekar, N., Chengalur, J., Subrahmanyan, R., \& Petitjean, P. 2001, A\&A, 367, 46

Kanekar, N., Smette, A., Briggs, F., \& Chengalur, J. 2009, ApJ 705,40

Lau, M. W., Prochaska, J., \& Hennawi, J. 2016, ApJS, submitted

Ledoux, C., Petitjean, P., Fynbo, J., Møller, P., \& Srianand, R. 2006, A\&A, 457, 71

Ledoux, C., Petitjean, P., \& Srianand, R. 2003, MNRAS, 346, 209

Lisenfeld, U., Espada, D., Verdes-Montenegro, L., et al. 2011, A\&A, 534, 102

McMullin, J. P., Waters, B., Schiebel, D., Young, W., \& Golap, K. 2007, in Astronomical Society of the Pacific Conference Series, Vol. 376, Astronomical Data Analysis Software and Systems XVI, ed. R. A. Shaw, F. Hill, \& D. J. Bell, 127

Møller, P., Fynbo, J., Ledoux, C., \& Nilsson, K. 2013, MNRAS, 430,2680

Muzahid, S., Srianand, R., \& Charlton, J. 2015, MNRAS, 448, 2840

Neeleman, M., Wolfe, A., Prochaska, J., \& Rafelski, M. 2013, ApJ, 769, 54
Noterdaeme, P., Ledoux, C., Petitjean, P., \& Srianand, R. 2008, A\&A, 481, 327

Petitjean, P., Théodore, B., Smette, A., \& Lespine, Y. 1996, A\&A, 313, L25

Prochaska, J., Chen, H.-W., Wolfe, A., Dessauges-Zavadsky, M., \& Bloom, J. 2008, ApJ, 672, 59

Prochaska, J., Weiner, B., Chen, H.-W., Mulchaey, J., \& Cooksey, K. 2011, ApJ, 740, 91

Richter, P., Krause, F., Fechner, C., Charlton, J., \& Murphy, M. 2011, A\&A, 528, 12

Saintonge, A., Kauffmann, G., Kramer, C., et al. 2011, MNRAS, 415,32

Sembach, K., Howk, J., Savage, B., \& Shull, J. 2001, AJ, 121, 992

Simcoe, R., Sargent, W., Rauch, M., \& Becker, G. 2006, ApJ, 637,648

Som, D., Kulkarni, V., Meiring, J., et al. 2015, ApJ, 806, 25

Stocke, J., Keeney, B., Danforth, C., et al. 2013, ApJ, 763, 148

Tremonti, C., Heckman, T. M., Kauffmann, G., et al. 2004, ApJ, 613,898

Tumlinson, J., Thom, C., Werk, J., et al. 2013, ApJ, 777, 59

Werk, J., Prochaska, J., Tumlinson, J., et al. 2014, ApJ, 792, 8

Wolfe, A., Prochaska, J., \& Gawiser, E. 2005, ARA\&A, 43, 861 